\title{
COVID-19, vulnerabilidade social e saúde mental das populações LGBTQIA+
}

\author{
COVID-19, social vulnerability and mental health \\ of LGBTQIA+ populations
}

\author{
COVID-19, vulnerabilidad social y salud mental de \\ las poblaciones LGBTQIA+
}

\author{
Geovani Bordiano 1 \\ Suzana Pacheco Liberal 1 \\ Giovanni Marcos Lovisi 1 \\ Lucia Abelha 1
}

doi: 10.1590/0102-311X00287220
Um importante aspecto observado durante a evolução da pandemia por COVID-19 é a relação entre vulnerabilidade social e maiores prevalência e letalidade do vírus 1. Os contrastes dessa mortalidade entre os mais pobres e mais ricos evidenciam como as desigualdades sociais impactam de forma direta a saúde da população brasileira e tornam mais vulneráveis determinados grupos sociais 1 .

Para além dos danos à saúde já conhecidos, a pandemia pode ser considerada um evento traumático e, assim, gerar graves consequências à saúde mental da população como um todo, tais quais insônia, depressão, reações de medo e raiva, abuso de substâncias, reações agudas ao estresse e transtorno do estresse pós-traumático 2 . Desse modo, a pandemia pode ser entendida como um catalisador para o adoecimento mental, especialmente para as populações mais vulneráveis.

As minorias sexuais e de gênero, ou seja, as pessoas lésbicas, gays, bissexuais, travestis, transexuais e transgêneros, queers, intersexuais, assexuais e outros (LGBTQIA+) sofreram, e ainda sofrem, um grave processo de exclusão social ao longo da história das sociedades contemporâneas 3 . Uma das consequências desse fato é a piora do estado de saúde mental desses grupos.

Aspectos como baixa escolaridade, baixo nível socioeconômico, violência e dificuldades de acesso aos serviços de saúde estão intimamente relacionados às minorias sociais e, ao mesmo tempo, têm sido considerados fatores de risco para transtornos mentais comuns na população em geral 4,5 . De forma adicional, outros fatores de risco à saúde mental são específicos desses grupos marginalizados: o preconceito (ou LGBTQIA+fobia) 6, a falta de proteção institucionalizada, a rejeição familiar e o bullying 7 . Toda essa conjuntura social coopera para a fragilização e vulnerabilidade dessas minorias.

Portanto, estudos nacionais e internacionais 8,9,10,11,12,13,14 revelam que as minorias sexuais e de gênero, quando comparadas à população não LGBTQIA+, possuem maiores prevalências de depressão e ansiedade, maior risco para o suicídio e fazem uso mais intenso de substâncias psicoativas.

\section{Pandemia, saúde mental e populações LGBTQIA+}

Em recente investigação nacional 13, realizada com cerca de 9 mil participantes, que objetivou averiguar os maiores impactos da pandemia para a população LGBTQIA+, 42,72\% dos entrevistados elencaram a saúde mental como o principal impacto vivenciado durante este período, seguida de "novas regras de convívio", "solidão", "convívio familiar" e "falta de emprego e dinheiro".
1 Instituto de Estudos em Saúde Coletiva, Universidade Federal do Rio de Janeiro, Rio de Janeiro, Brasil.

\section{Correspondência}

G. Bordiano Instituto de Estudos em Saúde Coletiva, Universidade Federal do Rio de Janeiro. Av. Horácio Macedo $s / n$, Rio de Janeiro, RJ 21941-598, Brasil.

giovannibordiano@gmail. com 
Dentre as razões que tornam os LGBTQIA+ mais vulneráveis em momentos como o atual, podemos citar os altos índices de violências a que estão submetidos cotidianamente. Apesar da carência de dados, números levantados pelo Instituto de Pesquisa Econômica Aplicada (IPEA) no Atlas da Violência 15 mostram um aumento de 127\% das denúncias de homicídio de LGBTQIA+ em 2018 no Brasil. Além disso, uma das mais importantes medidas sanitárias preconizadas pelos órgãos de saúde tem sido o distanciamento social que, no caso dos LGBTQIA+, pode significar permanecer confinado junto a agressores em potencial, frequentemente as suas próprias famílias ou parceiros íntimos 11,12,13,16.

$\mathrm{O}$ aumento da violência doméstica em decorrência das medidas de isolamento social tem sido trazido à tona nas discussões 17 , mas geralmente com foco exclusivo nas mulheres cisgênero heterossexuais. No entanto, é importante enfatizar que dentre os homicídios por LGBTQIA+fobia no Brasil, a residência da vítima é o segundo local de maior ocorrência de assassinatos 18 , sendo as pessoas transsexuais, transgêneros e travestis alvos preferenciais deste tipo de violência, seguidos das lésbicas e então dos homens gays 16 .

Outro fator de vulnerabilidade é o socioeconômico, permeando renda e vínculos trabalhistas. É sabido que a pandemia tem causado perda de empregos e precarização de vínculos empregatícios já frágeis, o que poderá agravar a situação socioeconômica dessas populações 11,12. Um estudo estadunidense 19 aponta que a maior parcela dessa população no país faz parte do mercado informal e também possui ocupações potencialmente mais expostas aos SARS-CoV-2, como trabalho em bares e restaurantes, além de terem menor renda e menores condições de acesso à saúde. No Brasil, quando se considera apenas as populações de transgêneros, transexuais e travestis, a vulnerabilidade é ainda maior dadas as especificidades destes grupos 12. Um estudo realizado com 672 transexuais no Estado de São Paulo revelou que, entre 2014 e 2015, apenas 16,7\% estavam no mercado formal 20 e, ainda, muitos trabalhavam como profissionais do sexo 21. Em relação à população homossexual e bissexual, uma revisão sistemática internacional 22 aponta que homens gays e bissexuais recebem menos que seus colegas heterossexuais. Além disso, a taxa de desemprego nessa população, encontrada por uma pesquisa 13 , é de $21,6 \%$ no Brasil e, durante a pandemia, 44,3\% sofreram uma paralisação total de suas atividades.

Outro importante ponto posto em fragilidade para os LGBTQIA+ durante a pandemia é a vida social. Como são por vezes rechaçados no núcleo familiar, um importante mecanismo de resistência e sobrevivência desses grupos está no vínculo que estabelecem com as suas comunidades, de forma que um grave impacto vivenciado por esses sujeitos está na impossibilidade, dadas as medidas de distanciamento social, de um contato mais ativo e presencial com seus afetos e territórios, o que leva a uma experiência de isolamento e solidão 11,12,13.

Diante de tantos pontos que comprometem a saúde mental das pessoas LGBTQIA+, torna-se indispensável que as autoridades definam estratégias de cuidado para este momento.

O Ministério da Saúde, em sua cartilha sobre suicídio na pandemia por COVID-19 23, elenca alguns fatores de alerta que merecem especial atenção, entre eles: problemas de saúde mental preexistentes, com destaque para a depressão e a ansiedade; uso de álcool e/ou outras drogas; ausência de apoio social; existência de hostilidade no ambiente familiar; e sofrimento e inquietações sobre a própria sexualidade. Em outra cartilha 24, que trata das recomendações para os gestores, o Ministério da Saúde ressalta que um dos pontos que deve receber atenção é a identificação dos grupos vulneráveis do ponto de vista psicossocial, e destaca diversas recomendações que podem ser instituídas pelos gestores durante a pandemia para garantir o acesso à saúde mental dessa população. Contudo, é notório que apesar de sua importância, a existência de instrumentos legais, de orientações e normativos, não é suficiente para a garantia do acesso da população aos seus direitos.

Não raras vezes esses grupos são expostos ao preconceito mesmo nos serviços de saúde, o que pode levar à diminuição da procura e da aderência a tratamentos 25,26. Assim, observa-se que a heteronormatividade presente nos diversos campos assistenciais produz uma desvalorização ou o não reconhecimento dessas identidades, contribuindo para a perpetuação de violências e discriminações que afetam as condições de acesso e qualidade da assistência à saúde 27 . Um estudo 27 relata que as principais dificuldades no acesso aos serviços de saúde para esse público estão relacionadas ao receio de sofrerem preconceitos e ao medo de revelar a orientação sexual, sendo esta informação muitas vezes omitida nos atendimentos. 
No Sistema Único de Saúde (SUS), a atenção especializada em saúde mental é realizada por meio da Rede de Atenção Psicossocial (RAPS), que encontra nos Centros de Atenção Psicossocial (CAPS) um de seus principais serviços 28 . No entanto, é importante enfatizar que a RAPS enfrenta obstáculos significativos, como insuficiência de equipamentos em algumas regiões e municípios brasileiros 29 , o que leva a longas filas de espera, e problemas de referência e contrarreferência 30. É notória, ainda, diante do grande percentual de discriminação sofrida em serviços de saúde, demonstrado em estudos 31, a carência de qualificação dos profissionais de saúde para lidarem com os sofrimentos transversais à sexualidade.

Considerando esses dados, é possível dizer que a saúde mental das minorias sexuais e de gênero carece, não apenas de atenção específica em termos de políticas públicas emergenciais de saúde durante a pandemia, mas, sobretudo, de mobilização e de ações concretas. Todavia, o que se atesta é uma profunda invisibilidade da questão por parte do Estado e, por vezes, também pela academia ${ }^{32}$, durante um momento de extrema necessidade. É urgente, além disso, que a discussão tome os espaços sociais e que a população LGBTQIA+ seja ouvida e vista, inclusive para que a situação atual sirva de lição para momentos futuros.

\section{Colaboradores}

G. Bordiano contribuiu com a concepção do projeto, análise e interpretação dos dados, redação, revisão crítica do conteúdo intelectual e aprovação da versão final a ser publicada e é responsável por todos os aspectos do trabalho. S. P. Liberal e G. M. Lovisi contibuíram com a concepção do projeto, análise e interpretação dos dados, redação, revisão crítica do conteúdo intelectual e aprovação da versão final a ser publicada. L. Abelha contribuiu com a revisão crítica do conteúdo intelectual e aprovação da versão final a ser publicada.

\section{Informações adicionais}

ORCID: Geovani Bordiano (0000-0002-32850689); Suzana Pacheco Liberal (0000-0001-98906895); Giovanni Marcos Lovisi (0000-0003-05210202); Lucia Abelha (0000-0002-2409-9872).

\section{Referências}

1. Fundação Oswaldo Cruz. Análise da frequência, incidência, mortalidade e letalidade por COVID-19 em favelas cariocas. Boletim Socioepidemiológio da COVID-19 nas Favelas 2020; (1). https://portal.fiocruz.br/sites/portal. fiocruz.br/files/documentos/boletim_socioe pidemiologicos_covid_nas_favelas_1.pdf.

2. Shigemura J, Ursano RJ, Morganstein JC, Kurosawa M, Benedek DM. Public responses to the novel 2019 coronavirus (2019-nCoV) in Japan: mental health consequences and target populations. Psychiatry Clin Neurosci 2020; 74:281-2.

3. Oliveira FAG, Carvalho HR, Jesus JG. LGBTI+ em tempos de pandemia da Covid-19. Diversitates International Journal 2020; 12:60-94.

4. Patel V, Kleinman A. Poverty and common mental disorders in developing countries. Bull World Health Organ 2003; 81:609-15.

5. Fundação Oswaldo Cruz. Saúde mental e atenção psicossocial na pandemia COVID-19: recomendações gerais. Rio de Janeiro: Fundação Oswaldo Cruz; 2020.

6. King M, Semlyen J, Tai SS, Killaspy H, Osborn D, Popelyuk D, et al. A systematic review of mental disorder, suicide, and deliberate self harm in lesbian, gay and bisexual people. BMC Psychiatry 2008; 8:70.

7. Shah R, Eshel N, McGlynn L. Lesbian, gay, bisexual, trasngender, and queer/questioning students. In: Roberts LW, editor. Student mental health: a guide for psychiatrists, psychologists, and leaders serving in higher education. Washington DC: American Psychiatric Association Publishing; 2018. p. 416-9.

8. Di Giacomo E, Krausz M, Colmegna F, Aspesi F, Clerici M. Estimating the risk of attempted suicide among sexual minority youths. JAMA Pediatr 2018; 172:1145-52. 
9. Teixeira-Filho FS, Rondini CA Suicide thoughts and attempts of suicide in adolescents with hetero and homoerotic sexual practices. Saúde Soc 2012; 21:651-67.

10. Remy LS, Scherer J, Guimarães L, Surratt HL, Kurtz SP, Pechansky F, et al. Anxiety and depression symptoms in Brazilian sexual minority ecstasy and LSD users. Trends Psychiatry Psychother 2017; 39:239-46.

11. OutRight Action International. Vulnerability amplified: the impact of the COVID-19 pandemic on LGBTIQ people. New York: OutRight Action International; 2020.

12. Carman M, Bourne A, Fairchild J. COVID-19 impacts for LGBTIQ communities and implications for services: a research briefing paper by Rainbow Health Victoria. Melbourne: Rainbow Health Victoria; 2020.

13. \#VOTELGBT. Diagnóstico LGBT+ na pandemia. https://www.votelgbt.org/pesquisas (acessado em Set/2020).

14. Francisco LCF, Barros AC, Pacheco M, Nardi AE, Alves V. Anxiety in sexual and gender minorities: an integrative review. J Bras Psiquiatr 2020; 69:48-56.

15. Cerqueira D, Bueno S, Lima RS, Neme C, Ferreira H, Alves PP, et al. Atlas de violência 2019. Brasília/Rio de Janeiro/São Paulo: Instituto de Pesquisa Econômica Aplicada/Fórum Brasileiro de Segurança Pública; 2019.

16. Pinto IV, Andrade SSA, Rodrigues LL, Santos MAS, Marinho MMA, Benício LA, et al. Perfil das notificações de violências em lésbicas, gays, bissexuais, travestis e transexuais registradas no Sistema de Informação de Agravos de Notificação, Brasil, 2015 a 2017. Rev Bras Epidemiol 2020; 23 Suppl 1:e200006.supl.1.

17. Vieira PR, Garcia LP, Maciel ELN. The increase in domestic violence during the social isolation: what does it reveals? Rev Bras Epidemiol 2020; 23:e200033.

18. Mendes WG, Silva CMFP. Homicide of lesbians, gays, bisexuals, travestis, transexuals, and transgender people (LGBT) in Brazil: a spatial analysis. Ciênc Saúde Colet 2020; 25:1709-22.

19. Whittington C, Hadfield K, Calderón C. The lives and livelihoods of many in the LGBTQ community are at risk amidst COVID-19 crisis. Washington DC: Human Right Campaign Foundation; 2020.

20. Silva MA, Carla GL, Veras MASM. Work and health issues of the transgender population: factors associated with entering the labor market in the state of São Paulo, Brazil. Ciênc Saúde Colet 2020; 25:1723-34.
21. Reidel M. A pedagogia do salto alto: histórias de professoras transexuais e travestis na educação brasileira [Dissertação de Mestrado]. Porto Alegre: Universidade Federal do Rio Grande do Sul; 2013.

22. Ozeren E. Sexual orientation discrimination in the workplace: a systematic review of literature. Procedia Soc Behav Sci 2014; 109:120315.

23. Fundação Oswaldo Cruz. Saúde mental e atenção psicossocial na pandemia de COVID-19: suicídio na pandemia de COVID-19. Rio de Janeiro: Fundação Oswaldo Cruz; 2020.

24. Fundação Oswaldo Cruz. Saúde mental e atenção psicossocial na pandemia de COVID-19: recomendações para gestores. Rio de Janeiro: Fundação Oswaldo Cruz; 2020.

25. Baptiste-Roberts K, Oranuba E, Werts N, Edwards LV. Addressing health care disparities among sexual minorities. Obstet Gynecol Clin North Am 2017; 44:71-80.

26. Dahlhamer JM, Galinsky AM, Joestl SS, Ward BW. Barriers to health care among adults identifying as sexual minorities: a US national study. Am J Public Health 2016; 106:1116-22.

27. Ferreira BO, Bonan C. Opening the closets of access and quality: an integrative review on the health of lgbtt populations. Ciênc Saúde Colet 2020; 25:1765-78

28. Lovisi GM, Abelha L, Schilling S, Muñoz RA. Prospects and challenges of mental health services in a major city in Latin America: Rio de Janeiro. In: Okkels N, Kristiansen CB, MunkJorgensen P, editors. Mental health and illness in the city. Singapore: Springer; 2017. p. 35775 .

29. Gama CAP, Guimarães DA, Coelho VAA, Carvalho RC, Campos CG, Fraga AMS. A implantação da rede de atenção psicossocial na Região Ampliada de Saúde Oeste de Minas Gerais BR. Cad Saúde Colet (Rio J.) 2020; 28:278-87.

30. Quinderé PHD, Jorge MSB, Franco TB. Rede de atenção psicossocial: qual o lugar da saúde mental? Physis (Rio J.) 2014; 24:253-71.

31. McClain Z, Thomas R, Yehia BR. Sociocultural and systemic barriers to health for gender and sexual minority populations. In: Smalley KB, Warren JC, Barefoot, Nikki K, editors. LGBT health: meeting the needs of gender and sexual minorities. New York: Springer; 2018. p. 16-8.

32. Bezerra MV, Moreno CA, Prado NM, Santos AM. Política de saúde LGBT e sua invisibilidade nas publicações em saúde coletiva. Saúde Debate 2019; 43(n.spe 8):305-23.
Recebido em 01/Out/2020

Versão final reapresentada em 25/Jan/2021

Aprovado em 12/Fev/2021 